\title{
Understanding of the performance of ARA antennas in ice
}

\author{
Simon Archambault* for the ARA Collaboration \\ Chiba University \\ E-mail: archs@chiba-u.jp
}

The Askaryan Radio Array (ARA) experiment uses radio antennas to detect the Askaryan emission from ultra-high-energy (above $10^{16} \mathrm{eV}$ ) neutrino interactions in the ice in Antarctica. The understanding of the antenna properties, such as the realized gain, is especially crucial for the energy determination of neutrino events, and the performance has to be optimized for the maximum detection efficiency.

The understanding of the performance in ice, on the other hand, is challenging. In order to understand the performance in ice, the calibration in ice was performed at the South Pole, in parallel with antenna simulations in ice. We performed the study using a new slim version of ARA antennas which could be used for future deployment, with which the installation would be much faster and cost effective.

35th International Cosmic Ray Conference - ICRC2017

10-20 July, 2017

Bexco, Busan, Korea

\footnotetext{
* Speaker.

${ }^{\dagger}$ https://ara.wipac.wisc.edu
} 


\section{Introduction to ARA}

The Askaryan Radio Array (ARA) experiment aims at detecting neutrinos coming from ultrahigh-energy cosmic rays (UHECRs) interacting with the cosmic microwave background through the Greisen-Zatsepin-Kuzmin (GZK) effect [1, 2, 3]. The resulting neutrinos would then interact in the Antarctic ice sheet, culminating in Askaryan radio emissions [4, 5].

ARA is currently under construction at the geographic South Pole, next to the IceCube experiment. The current plan is to build 37 antenna clusters, or stations, in a hexagonal grid, as shown in the left image of Figure 1. The right image shows the layout of an ARA station, with four strings, each equipped with four antennas, two for each polarization (vertical and horizontal). The two polarizations are used to improve neutrino reconstruction. Each station is separated by $2 \mathrm{~km}$ spacing, to maximize the experiment's effective area at $10^{18} \mathrm{eV}[6]$.
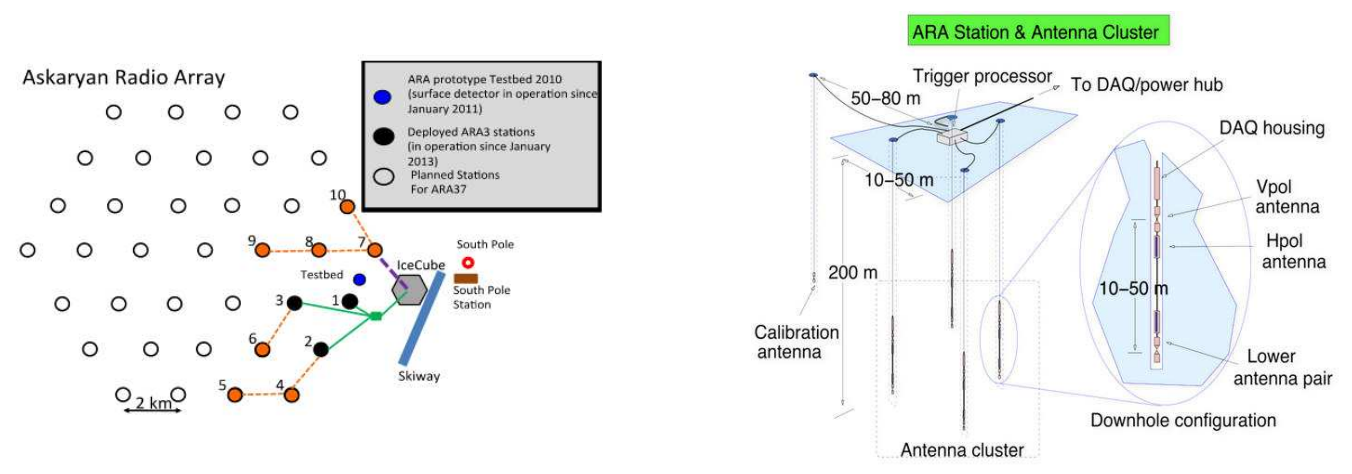

Figure 1: Left: Schematic of the planned layout of ARA, at the South Pole. The black filled circles are the stations already installed, while the black hollow circles are the projected stations. Right: Design of an ARA station, the 4 strings containing 2 HPol and 2 VPol antennas, as well as accompanying instrumentation.

These proceedings focus on the development and understanding of the properties of the ARA antennas. Understanding their behavior in ice is necessary for proper reconstruction of the events. The next sections will outline the different steps taken to understand this behavior, and use these findings to optimize the antenna design.

\section{Antenna Design}

\subsection{Original ARA Antennas}

Due to the sizes of the holes in the Antarctic ice being limited to a diameter of $15 \mathrm{~cm}$, ARA antennas need to be less than $15 \mathrm{~cm}$ wide. Other design constraints require a hole in the middle of the antennas so the cables can go through all the way, connecting top antennas to bottom ones.

The VPol antennas are birdcage dipoles (see left image of Figure 2) of a diameter of $13.9 \mathrm{~cm}$; the center of which is hollow, and the feed region is annular around the cables. The feed is connected to the cable at multiple locations along the annulus, using a crossbar connection (as can be seen in the left image of Figure 6). The properties of these antennas, such as the realized gain, will be explored in Section 4.

Designing proper $\mathrm{HPol}$ antennas, covering a frequency range down to $150 \mathrm{MHz}$ proved to be very challenging. The final design used slotted copper cylinders loaded with internal ferrites to lower 
its frequency response. The right image of Figure 2 shows a picture of this HPol design. It has a diameter of $12.7 \mathrm{~cm}$. More information on the antenna designs can be found in [6].

\subsection{Development of Slim Antennas}

ARA currently uses a hot-water drill to bore holes in the ice, which allows to dig deep dry holes. However, it has a few prohibitive features. It is expensive to operate, requires large manpower, and it takes $\sim 10$ hours to drill a single hole. In the hopes of solving these problems, a new drill has been developed: The Rapid-Air-Movement (RAM) drill ${ }^{1}$. This drill uses high-velocity air to rotate cutters and blow ice chips from the hole. This drill is much cheaper, and much faster to use (allowing to drill $\sim 20$ holes per day). However, this drill can only make a hole of $10 \mathrm{~cm}$ diameter, and only up to $100 \mathrm{~m}$ deep. In order to explore the possibility of using this drill, a slimmer version of the ARA antennas described previously were developed. They follow the same design as the original model, with a reduced diameter of $7.9 \mathrm{~cm}$. Figure 2 shows a side-by-side comparison of the two antenna models for VPol and HPol antennas.
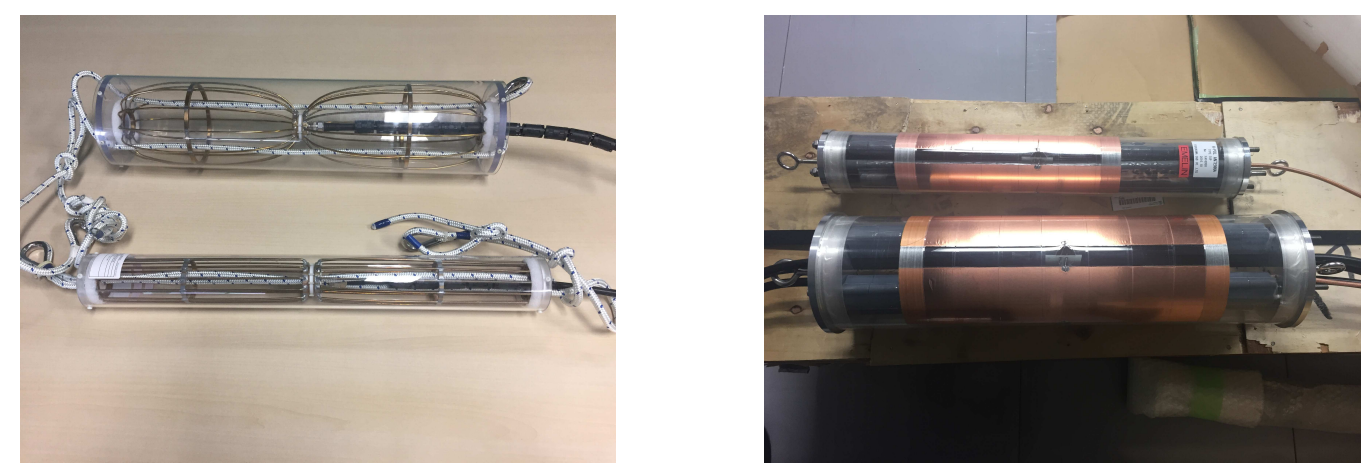

Figure 2: Side-by-side comparison of slim and large antennas developed by ARA. Left image shows the VPol antennas, while the right image shows the HPol.

The development of these antennas is for exploratory purposes only. A deeper deployment than what it allows is preferable due to the effect of the changing index of refraction in the top $200 \mathrm{~m}$ of the ice [6]. Antennas in shallow ice would in effect reduce the overall effective area of the experiment. The cheaper cost, however, allows to bore more holes and compensate this loss. As it stands now, though, the original antennas are the favored choice for the near future. However, the slim antennas are mentioned here as these antennas design were used in January 2017 at the South Pole for the measurement discussed in the next Section.

\section{South Pole Measurement}

\subsection{Purpose of Measurement}

In order to properly reconstruct the events seen by ARA as well as to understand the behavior of the detector, it is important to understand the behavior of the antennas themselves. The main quantity used is the realized gain, or the ability of the antennas to transfer an electrical pulse into a change in the electric field (i.e. a radio pulse) or vice-versa. Generally, the realized gain should be as high as possible.

\footnotetext{
${ }^{1}$ http://icedrill.org/equipment/ram.shtml
} 
It is relatively easy to characterize antennas in air, at room temperature. Section 4 will describe these methods. However, the characterization in the South Pole ice, in cold temperatures, is more challenging. Putting the antenna at low temperatures is not enough, as most of the effects will be due to the ice's index of refraction (which also changes as function of depth [6]). Slim antennas were therefore taken to the South Pole and placed the ice, in one of the boreholes already dug by the RAM drill. In this way, the realized gain and VSWR of the antennas in the South Pole ice could be measured.

\subsection{Methodology}

Two holes were used, separated by $20 \mathrm{~m}$. One antenna was placed in one of the two holes. This one was allowed to move in depth, between 5 and $35 \mathrm{~m}$. This antenna was used as the transmission antenna, and was connected to a network analyzer at the surface. In the other hole, another antenna was used, placed and fixed at a depth of $10 \mathrm{~m}$. This antenna was used as a receiver. A measurement was carried using two VPol antennas, and another using two HPol antennas. The receiving antenna was connected to a low-noise amplifier (LNA), a device designed to amplify the signal in the 100 to $900 \mathrm{MHz}$ region, where most of the Askaryan signal is expected [6]. All of this was connected at the surface to a $26 \mathrm{~dB}$ amplifier and to another port on the network analyzer. The network analyzer was used to measure $S_{21}$ (the ratio of the power received at Port 2 with respect to the power sent from Port 1) as well as the VSWR. To get a sense of the antenna's angular response in ice as well as the effect of the changing index of refraction, the measurement was taken with the transmitting antenna at depths of 5, 15 and $36 \mathrm{~m}$ (for VPol) and 5, 15, 26 and 35m (for HPol). Figure 3 shows a schematic of the setup used for this measurement.

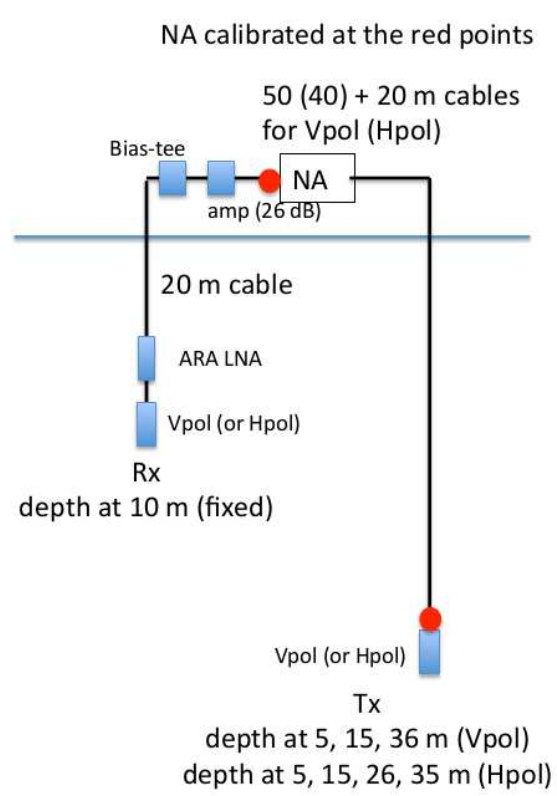

Figure 3: Configuration for the South Pole antenna gain measurements. Note that the red points indicate the points where the network analyzer was calibrated.

\subsection{Analysis and Results}

The analysis uses the Friis transmission equation [7] to calculate the realized gain of the antennas:

$$
S_{21}=\frac{P_{r}}{P_{t}}=G_{t} G_{r}\left(\frac{\lambda}{4 \pi R}\right)^{2}
$$

where $P_{r}$ and $P_{t}$ are the power received and transmitted (their ratio being the S21 value as measured by the network analyzer), $G_{t}$ and $G_{r}$ are the gain of the transmitted and of the receiving antenna respectively, $\lambda$ is the wavelength, and $R$ is the distance between the antennas. As the 2 antennas can be assumed to be identical, $G_{t}$ and $G_{r}$ can be replaced with $G^{2}$.

When doing the analysis, it is important to remove the effect of the different components, i.e the 
LNA $\left(S_{21}^{L N A}\right)$, the amplifier $\left(S_{21}^{a m p}\right)$ and the cables $\left(S_{21}^{c a b l e}\right)$. They are measured separately using the network analyzer. Using this knowledge, Equation 3.1 looks like this:

$$
S_{21}^{t o t}=\frac{P_{r}}{P_{t}}=G^{2} S_{21}^{L N A} S_{21}^{A m p} S_{21}^{\text {cables }}\left(\frac{\lambda}{4 \pi R}\right)^{2}
$$

The realized gain can then be found by isolating $G$. The resulting gain is shown in Figure 5, compared with results from simulations (explained in the next Section). Some fluctuation is visible, which is not seen in simulations and is still under investigation.

\section{Comparing with Simulations}

\subsection{Simulation Software}

The realized gain of the antennas themselves are needed when simulating an experiment like ARA, as a component needed to predict the antennas' reactions to an Askaryan signal. This is done using a simulation package, specialized in simulating the behavior of antennas. XFDTD ${ }^{2}$ is a simulation software that uses the finite-difference-time-domain algorithm [8] to accomplish this. The design of the antennas is given to the software, and a pulse is sent at the antenna feed. The simulation propagates the signal through the antenna and then propagates the emitted electric field in the surrounding volume. It does this by breaking down the antenna and surrounding volume in a grid, where Maxwell's Equations are solved in each of its cells. This algorithm then leads to the computation of quantites pertinent to describe antenna properties, such as $S_{11}$ or the realized gain.

\subsection{Comparing with Anechoic Chamber Data}

Measurement data is needed to confirm the behaviour of the antenna simulations. A solution is to go in an anechoic chamber to take the measurement. An anechoic chamber is a room that is RF-isolated from the outside world, and minimizes effects from random radio wave interference. Measuring the antenna gain in an anechoic chamber proceeds in two steps, using three antennas and a network analyzer. A schematic of the setup is shown in Figure 4. At first, the network analyzer measures $S_{21}$ using 2 antennas as a cal-

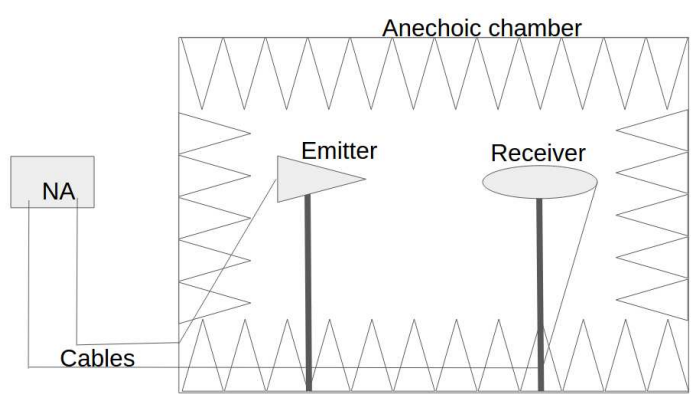

Figure 4: Schematic of the setup inside an anechoic chamber. ibration measurement $\left(S_{21}^{R e f}\right)$, one emitter that does not change and a reference receiver with a known gain $\left(G_{R e f, k n o w n}\right)$. Then, the antenna-under-test (AUT) replaces the reference antenna, making sure that the position of the AUT is exactly the same as that of the reference antenna during its measurement. The network analyzer records the $S_{21}$ for that setup as well $\left(S_{21}^{A U T}\right)$. Then, the realized gain of the AUT is found this way:

$$
G_{A U T}(d B)=S_{21}^{A U T}(d B)-S_{21}^{R e f}(d B)+G_{R e f, k n o w n}(d B)
$$

\footnotetext{
${ }^{2}$ https://www.remcom.com/xfdtd-3d-em-simulation-software/
} 
This measurement was done in an anechoic chamber available at Chiba University. Results from these measurements are shown in the left plot of Figure 5, with the VPol antennas, alongside the corresponding simulation results from XFDTD, made in air, to match with the measurement. The measurement shows a realized gain matching the prediction from the simulation (in vacuum $(n=1)$, which is very close to air $(n=1.00029$ at STP), despite large fluctuations especially at the low-frequency range.
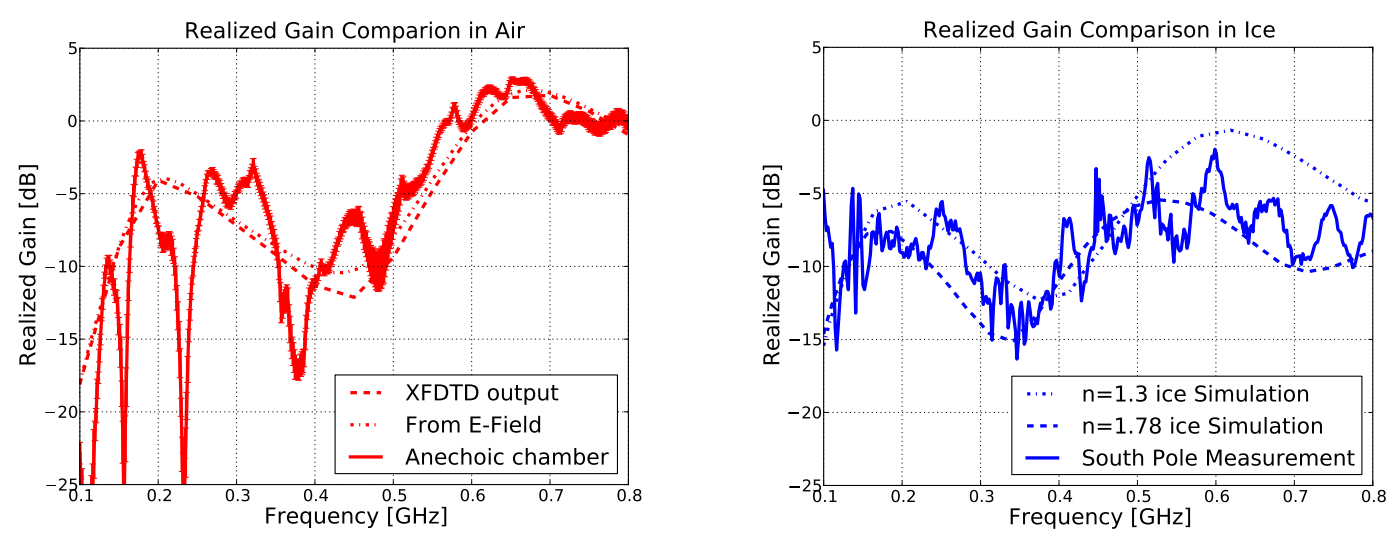

Figure 5: Left: Measurements made in anechoic chamber (full line) compared to simulations in vacuum. Two simulations are shown, the XFDTD output (dashes), and the calculated one (dashdotted). Right: South Pole measurements (full line) compared with simulations in ice, of $n=1.3$ (dash-dotted) and $n=1.78$ (dashes). Note that both show the gain of VPol antennas, when measured at an inclination of 55 degrees from the normal of the antenna.

\subsection{Developing in-ice Simulations}

A naive approach to simulate antennas in ice is to place the antenna in a cube of ice, and launch the simulation. However, the software will automatically calculate the realized gain from outside the cube of ice. The overall realized gain then takes into account the interface between ice and vacuum (as the overall material of the software cannot be set to anything but vacuum).

A solution is to calculate the realized gain by hand, by using other outputs that XFDTD provides. In order to do this, XFDTD needs to propagate the EM wave all the way to the far-field, which is used to calculate the realized gain. Normal FDTD algorithms take the near-field and propagate it into the far-field, once the near-field satisfies convergence criterias. This propagation is done without having to compute Maxwell's equation in each cell of the grid up to the far-field region, which would otherwise require a large amount of computer memory. This method will not work in ice, as the calculations will have to be made for each cell in the ice anyway, and the propagation to the far-field would take place after the EM wave left the cube. To avoid this, the cube of ice can be made to be as large as possible so that it reaches the far-field region. This, however, is limited by the amount of computer memory available, as now the electric field has to be calculated for each cell in the grid up to the edge of the cube, in the far-field.

One way to limit this problem is to make the cube as large as the computer memory will let it, and take the electric field near its edge as being in the far-field. Using this electric field with the other outputs provided by XFDTD (i.e. input voltage, $S_{11}$ ), the realized gain can be calculated. The results of simulations provided here are calculated using an electric field at a distance of $2.8 \mathrm{~m}$, 
with the antenna placed at the center of a cube of ice $6 \mathrm{~m}$ large. These distances are not perfect to be considered far-field, but still work. Realized gain using this method in a cube of air is compared with the actual result from XFDTD (shown in the left plot of Figure 5), and shows that the result agree well, particularly in the high-gain regions ( $\operatorname{sim} 0.2 \mathrm{GHz}$ and above $0.5 \mathrm{GHz}$ ), while the disagreement is $\sim 1 \mathrm{~dB}$ in the lowest-gain regions (around $0.4 \mathrm{GHz}$ ), for a total discrepancy of $\sim 8 \%$ when comparing the integrated gain in the frequency range of interest. It is likely a similar discrepancy will be present in the in-ice simulations. Given the uncertainties and fluctuations in the in-air measurement shown here, this difference is acceptable.

\subsection{Comparing with South Pole Measurements}

Results of these simulations are shown in Figure 5, using ice with an index of refraction of 1.3 (i.e. ice near the surface) as well as 1.78 (ice at $200 \mathrm{~m}$ depth). Since the ice's index of refraction changes progressively with depth, it is expected that the simulations do not match completely with the data, instead providing 'bounds' between the shallow and deep ice. However, the results suggest the simulations' outputs are believable.

\section{Improving antenna design}

As antenna simulations in ice are becoming better understood, ARA is in a better position to investigate the antenna design and optimize it.

\subsection{Modifying the Antenna Feed}

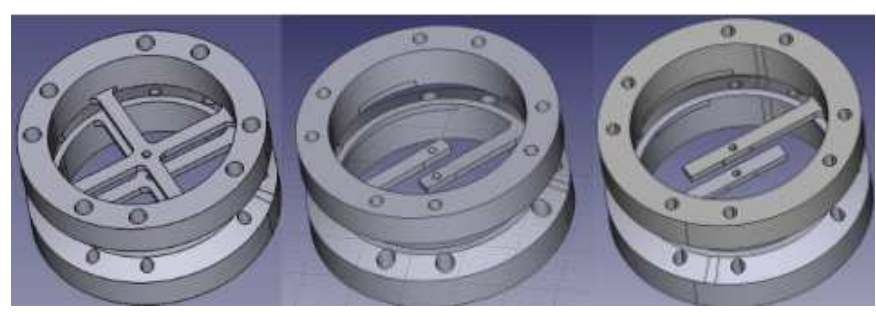

Figure 6: Picture showing the crossbar (left), half bar (middle) and three-quarter bar (right) feeds investigated here.
One of the aspects to look into is the antenna feed itself. The original antenna design uses a flat plate feed. Slim antenna models use a crossbar feed, and other feeds being explored are the use of a single bar feed, but not going through the whole diameter, only stopping half way (a half-bar feed), or a similar design with a bar stopping $3 / 4$ of the way (three-quarter feed). Figure 6 shows an image of the different feeds, while the left plot of Figure 7 shows their respective realized gain from simulation, and the right plot shows their angular pattern. This suggests an improvement using the 3/4 feed, at least at $400 \mathrm{MHz}$. The overall effect on the experiment's sensitivity still needs to be confirmed, by looking at the improvement on ARA's effective area using a simulation software developed by the collaboration (ARASim) [9].

\section{Summary}

Using the XFDTD software, it is possible to get antenna properties such as the realized gain from simulations, either in air or in ice. The comparison of the gain's behavior between ice and air will then be used to estimate the antenna gain in ice based on measurements in anechoic chamber. This allows us to use the measured realized gain in ARA simulations, without having to take measurements in the Antarctic ice, which presents a logistical challenge. Finally, these simulations can 

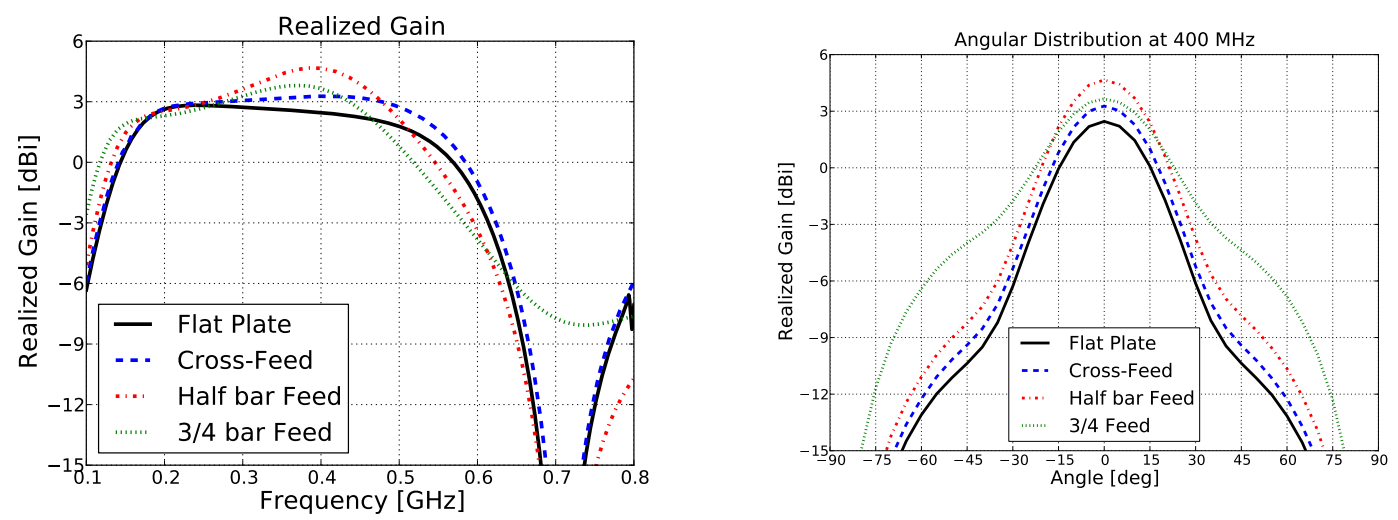

Figure 7: Realized gain (Left) and angular pattern (Right) of VPol antennas with different feed types. The realized gain is shown when facing the antennas, in ice, while the angular distribution looks at the behavior at $400 \mathrm{MHz}$.

be used to investigate improvement in antenna design, to improve the sensitivity of the experiment. A few changes are being explored with regards to the feed component of the antennas, and work is in progress to determine the overall effect in ARA's effective area.

\section{References}

[1] K. Greisen. End to the Cosmic-Ray Spectrum?, PRL 16, 748, 1966.

[2] G.T. Zatsepin and V.A. Kuzmin Upper limit of the spectrum of cosmic rays, JETP Letters 4, 78, 1966.

[3] V.S. Beresinsky and G.T. Zatsepin Cosmic rays at hultra high energies (neutrino?), Phys. Lett. B 6, 423, 1969.

[4] G.A. Askaryan. Excess Negative Charge of an Electron-Photon Shower and its Coherent Radio Emission, JETP 41, 616, 1962.

[5] G.A. Askaryan. Coherent Radio Emission from Cosmic Showers in Air and in Dense Media, JETP 48, 988, 1965.

[6] P.Allison, J. Auffenberg, R. Bard et al. Design and initial performance of the Askaryan Radio Array prototype EeV neutrino detector at the South Pole, Astroparticle Physics 35, 457, 2012.

[7] H.T. Friis A Note on a Simple Transmission Formula, Proceedings of the IRE 34, 254, 1946

[8] D.M. Sullivan Electromagnetic Simulation using the FDTD Method, IEEE Press Series on RF and Microwave Technology, Piscataway, 2000.

[9] P. Allison, J.Auffenberg, R. Bard et al. First constraints on the ultra-high energy neutrino flux from a prototype station of the Askaryan Radio Array, Astroparticle Physics 70, 62, 2015. 